\title{
Insulin-like growth factor-1 levels predict myocardial injury and infarction after elective percutaneous coronary intervention: an optical coherence tomography study
}

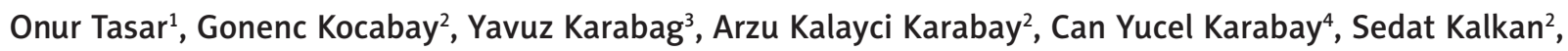 \\ Cevat Kirma
}

${ }^{1}$ Department of Cardiology, Elazig Research and Training Hospital, Elazig, Turkey

${ }^{2}$ Department of Cardiology, Kartal Kosuyolu Heart and Research Hospital, Istanbul, Turkey

${ }^{3}$ Department of Cardiology, Kars Kafkas University Medicine, Faculty Hospital, Kars, Turkey

${ }^{4}$ Department of Cardiology, Siyami Ersek Heart Center, Istanbul, Turkey

Adv Interv Cardiol 2020; 16, 2 (60): 162-169

DOI: https://doi.org/10.5114/aic.2020.96059

\section{A bstract}

Introduction: Periprocedural myocardial necrosis, which can range from a low level elevation of cardiac biomarkers to a large myocardial infarction (MI), is a common complication after percutaneous coronary intervention (PCI).

Aim: We hypothesized that insulin-like growth factor-1 (IGF-1) levels may play a protective role in myocardial injury after coronary stent placement and aimed to investigate the relationship between IGF-1 levels and plaque characteristics assessed by optical coherence tomography (OCT).

Material and methods: Between May 2015 and December 2015 we prospectively enrolled 74 patients with stable angina pectoris in whom single de novo coronary artery stenosis was present. PCI was performed according to standard methods. OCT was applied to all patients. TnT was analyzed at admission, before PCl and at 6, 12, 24 and $48 \mathrm{~h}$ after PCI. Serum IGF-1 was measured prior to $\mathrm{PCl}$.

Results: A total of 25 (33.7\%) patients had periprocedural myocardial injury or type 4a myocardial infarction, and 49 (66.2\%) patients had no events. IGF-1 level and reference intimal thickness, medial thickness, and plaque fibrous cap thickness in OCT had strong correlations ( $r=0.88,0.80$ and 0.88 respectively, $p<0.001)$. IGF-1 was an independent predictor of periprocedural myocardial injury or type $4 \mathrm{a} \mathrm{MI}$ in univariate $(\mathrm{OR}=0.929,95 \% \mathrm{Cl}: 0.895-0.964, p<0.001)$ and multivariate regression analysis $(\mathrm{OR}=0.757$, $95 \% \mathrm{Cl}: 0.575-0.998, p=0.04)$. Based on ROC analysis, the best cut-off value of IGF-1 for predicting periprocedural myocardial injury or type 4 a myocardial infarction was $144.5 \mathrm{ng} / \mathrm{ml}$, with a maximum sensitivity of $88 \%$ and specificity of $77.6 \%$ (AUC $=0.80$, $95 \%$ Cl: $0.69-0.88, p<0.0001)$.

Conclusions: The results from this study indicate that low IGF-1 levels are associated with plaque instability assessed by OCT. Low IGF-1 levels may identify patients who are at increased risk for periprocedural myocardial injury/infarction.

Key words: insulin-like growth factor-1 levels, myocardial injury, percutaneous coronary intervention.

Su m m a ry

This study may elucidate the mechanism responsible for periprocedural myocardial injury/infarction in the presence of a low level of insulin-like growth factor-1.

\section{Introduction}

Periprocedural myocardial necrosis, which can range from a low-level elevation of cardiac biomarkers to a large myocardial infarction (MI), is a common complication after percutaneous coronary intervention $(\mathrm{PCI})$. While ad- vances in interventional technology and techniques have translated into a reduction in procedure-related morbidity and mortality, the incidence of procedural myocardial injury/infarction has not changed significantly [1]. However, MI associated with percutaneous coronary interven-

\section{Corresponding author:}

Gonenc Kocabay MD, Cardiology Department, Kartal Kosuyolu Heart and Research Hospital, Istanbul, Turkey, phone: +90 5325180035 , e-mail: gonenckocabay@yahoo.com

Received: 4.12.2019, accepted: 30.03.2020. 
tion (type 4a MI) is defined by a post-procedural rise of troponin values to five times the $99^{\text {th }}$ percentile upper rate limit (URL) in patients with normal baseline values, or a rise in troponin values by $20 \%$ if the baseline values are elevated but are stable or falling. The Universal Definition further mandates that the post-procedural elevation of troponin should be accompanied by clinical, electrocardiographic (ECG), echocardiographic or angiographic evidence of myocardial ischaemia.

Insulin-like growth factor-1 (IGF-1) is important for the plaque stability in atherosclerosis due to its positive effects on vascular smooth muscle cells (VSMC) [2]. Since IGF-1 plays an important role in VSMC apoptosis and migration, low levels may play an important role in acute coronary syndrome by promoting plaque instability [3]. Moreover, it was shown that IGF-I is involved in the repair of the intima in injured arteries. Smooth muscle cell accumulation is important for the neointimal proliferation after angioplasty. Hence, IGF-1 can be effective for neointimal proliferation after angioplasty [4].

Optical coherence tomography (OCT) is an intravascular imaging method that demonstrates the detailed visualization of plaque components and morphology and is used for the optimization of procedural outcome [5]. OCT allows for accurate determination of fibrous cap thickness, thereby enabling in vivo identification of the presence of thin-cap fibroatheroma (TCFA) [6]. The presence of a thin fibrous cap or increased lipid core on intravascular imaging is a predictor of a periprocedural $\mathrm{MI}$. OCT-based fibrous cap thickness is the most important predictor of periprocedural MI [7].

\section{Aim}

In the present study, we hypothesized that IGF-1 levels may play a protective role in myocardial injury after coronary stent placement and aimed to investigate the relationship between IGF-1 levels and plaque characteristics assessed by OCT.

\section{Material and methods}

\section{Study population}

Between May 2015 and December 2015 we prospectively enrolled 74 patients with stable angina pectoris in whom single de novo coronary artery stenosis was present. We performed PCI in the case of lesions described as significant stenosis. Significant stenosis was defined as angiographic > 50\% diameter stenosis in patients with ischemia that was detected during myocardial perfusion scintigraphy or the treadmill exercise test. Stenosis $>50 \%$ in the absence of ischemia testing was defined as significant if the fractional flow reserve (FFR) value of the stenosis was $<0.80$. Patients with acute coronary syndrome or elevated high-sensitivity troponin T (TnT) level at admission were excluded. Also, previous coronary artery bypass grafting, patients with bifurcation lesions and previous myocardial infarction, lesions involving the left main stem and chronic total occlusions were excluded from the study to take optimal OCT measures. Patients with an estimated glomerular filtration rate below $60 \mathrm{ml} /$ min (by the Cockcroft and Gault formula) and use of insulin were excluded as well. All type 2 diabetic patients were on metformin treatment. All patients provided written informed consent and the study was approved by the institutional review board.

\section{Clinical and laboratory data}

All clinical features of the patients were collected at admission. Creatinine, glycated hemoglobin $\left(\mathrm{HbA}_{1 \mathrm{c}}\right)$ and lipid profile were determined by standard methods. The assay used in our center measures cardiac troponin T (cTnT) with high sensitivity using the immuno-analysis ECL-sandwich by Roche. The lower detection limit was $0.01 \mathrm{ng} / \mathrm{ml}\left(99^{\text {th }}\right.$ percentile of reference control group). TnT was analyzed at admission, before $\mathrm{PCl}$ and at 6, 12, 24 and $48 \mathrm{~h}$ after $\mathrm{PCl}$. For IGF-1, at the baseline examination, $3 \mathrm{ml}$ of serum from each participant was obtained from subjects in a supine, nonfasting state in the afternoon and stored at $-80^{\circ} \mathrm{C}$. Serum IGF-1 was measured by radioimmunoassay after acid-ethanol extraction in 3 batches16; the intra-assay coefficient of variation was $<4 \%$. Insulin-like growth factor binding protein levels were not measured. The assay used in our center measures TnT with high sensitivity using the immuno-analysis (ECL-sandwich by Roche). According to the fourth universal definition of myocardial infarction [8]; cardiac procedural myocardial injury was defined by increases of cTn values ( $>99^{\text {th }}$ percentile URL) in patients with normal baseline values $\left(\leq 99^{\text {th }}\right.$ percentile URL) or a rise of cTn values $>20 \%$ of the baseline value when it is above the $99^{\text {th }}$ percentile URL but it is stable or falling; and coronary intervention-related MI was defined by an elevation of cTnT values more than five times the $99^{\text {th }}$ percentile URL in patients with normal baseline values. In patients with elevated pre-procedure cTn in whom the cTnT level is stable ( $\leq 20 \%$ variation) or falling, the post-procedure cTnT must rise by $>20 \%$. However, the absolute post-procedural value must still be at least five times the $9^{\text {th }}$ percentile URL. In addition, one of the following elements is required: new ischaemic ECG changes; development of new pathological Q waves; imaging evidence of new loss of viable myocardium or new regional wall motion abnormality in a pattern consistent with an ischaemic etiology; angiographic findings consistent with a procedural flow-limiting complication such as coronary dissection, occlusion of a major epicardial artery or a side branch occlusion/thrombus, disruption of collateral flow, or distal embolization.

\section{PCI procedure}

$\mathrm{PCl}$ was performed according to standard methods, and images were stored digitally. $300 \mathrm{mg}$ aspirin, $600 \mathrm{mg}$ 
loading and 75 mg daily clopidogrel doses were administered to all study patients. Glycoprotein Ilb/IIla inhibitors were used in 1 patient who developed no-reflow. All patients received weight-adjusted intravenous unfractionated heparin. To all patients before and after $\mathrm{PCl}$, OCT was applied in the culprit vessel only, specifically for this study. To obtain images of basal lesions, a C7XR LightLab Dragonfly OCT catheter (St. Jude Medical, Little Canada, MN, USA) with an automatic motorized pullback system $(20 \mathrm{~mm} / \mathrm{s})$ was used after administering $200 \mu \mathrm{g}$ of intracoronary nitroglycerine. The catheter was advanced distally to the lesion over a conventional 0.014-inch guidewire and images were obtained nonocclusively by motorized pullback at $20 \mathrm{~mm} / \mathrm{s}$ during $4 \mathrm{ml} / \mathrm{s}$ flushing with a total of $14-16 \mathrm{ml}$ of contrast media, Visipaque (GE Health Care, Cork, Ireland) or Iomeron (Ilsung Corp., Seoul, Korea). Prior to predilatation, stent diameter and length were determined in accordance with OCT images and at the discretion of the primary operator. All patients received a Xience pro (Abbott Vascular) stent implanted with $14 \mathrm{~atm}$ pressure. OCT images were acquired immediately using the same catheter following stent implantation. Afterwards, either termination of the procedure, the requirement of postdilatation with a noncompliant balloon, or additional stent implantation was decided in accordance with the last OCT images, angiogram, and at the discretion of the primary operator. Following this, once again OCT acquisition was taken for stent optimization and index parameters in cases of additional stent implantation and/or noncompliant balloon dilation. In the presence of stent edge dissection in only OCT images without angiographic evidence, an additional stent was not implanted. No-reflow was defined as thrombolysis in myocardial infarction $(\mathrm{TIMI})<3$ distal flow at the index procedure. Hemodynamic complications during the procedure were defined as hypotension and dysrhythmia, and angiographic complications were defined as any coronary dissection, no-reflow or vessel rupture.

\section{OCT quantitative analysis}

Optical coherence tomography quantitative analysis was performed by an independent observer using the C7-XR FDOCT system (St. Jude Medical). The system was calibrated for z-offset and images were analyzed frame by frame. Lesion lengths and stent lengths were measured in longitudinal images by the device in a St. Jude Workstation. On each OCT frame within the stent, all struts were counted and each strut was evaluated for stent apposition. Malapposition was defined in cases where the distance from the surface of the strut to the lumen contour was longer than the implanted stent strut thickness. A consensus of 2 independent observers for each frame was obtained. Cross-sectional OCT images were analyzed frame by frame. Cholesterol crystal was defined as a thin linear region of high signal intensity within a lipid plaque [9]. Stent edge dissection was defined as disruption of the luminal vessel surface in the edge segments within $5 \mathrm{~mm}$ proximal and distal to the stent while no struts are visible [10]. Lipid plaque was defined as a signal-poor region with diffuse borders, calcified plaque as a well-delineated, signal-poor region with sharp borders, and fibrous plaque as a homogeneous signal-rich region [11]. Plaque type at a patient level was defined according to the lesion which is causing only critical stenosis. Plaque length was measured by ruler in longitudinal images. The plaque arc of the most critical section of each lesion was measured manually by an electronic goniometer in cross sectional images. Lipid volume index was defined as the mean lipid arc multiplied by lipid length [12]. Reference intimal thickness, reference medial thickness, and plaque fibrous cap thickness were measured by ruler in cross-sectional images (Figure 1). Minimal lumen area (MLA) was defined as the smallest lumen area along the length of the target lesion and reference lumen area (RLA) was defined as the site with the largest lumen either nearest proximal or distal to the stenosis within the same segment. Percent luminal area stenosis was defined as the relative decrease in the luminal area of the target lesion when compared with the reference lumen in the same vessel segment. These measurements were calculated by the device automatically. Minimal lumen diameter (MLD) was defined as the smallest lumen diameter along the length of the target lesion and reference lumen diameter (RLD) was defined as the site with the largest lumen diameter either nearest proximal or distal to the stenosis within the same segment [9]. Post-stent luminal mass was defined as the presence of thrombus or plaque materials in the lumen after stent implantation in OCT images [13]. Spotty calcification was defined as the presence of lesions $<4 \mathrm{~mm}$ in length and containing an arc of calcification $<90^{\circ}$ in OCT images [14]. Thrombus was defined as an irregular mass with minimum diameter at least $250 \mu \mathrm{m}$ adherent to the vessel wall or floating within the lumen in OCT images. Microvessels within the intima in plaque were defined as signal-poor voids that are sharply delineated and usually can be followed in multiple contiguous frames. Plaque rupture was defined as a fibrous cap discontinuity with cavity formation. Macrophages were defined as signal-rich, distinct, or confluent punctate regions that exceed the intensity of background speckle noise. Calcified nodules in OCT (a calcific nodule is defined as a single or multiple regions of calcium (defined previously) were defined as masses protruding into the lumen that frequently form sharp and jutting angles. An OCT thin-capped fibroatheroma (OCT-TCFA) was defined as an IVOCT-delineated necrotic core with an overlying fibrous cap where the minimum thickness of the fibrous cap is less than $65 \mu \mathrm{m}$ [9]. 

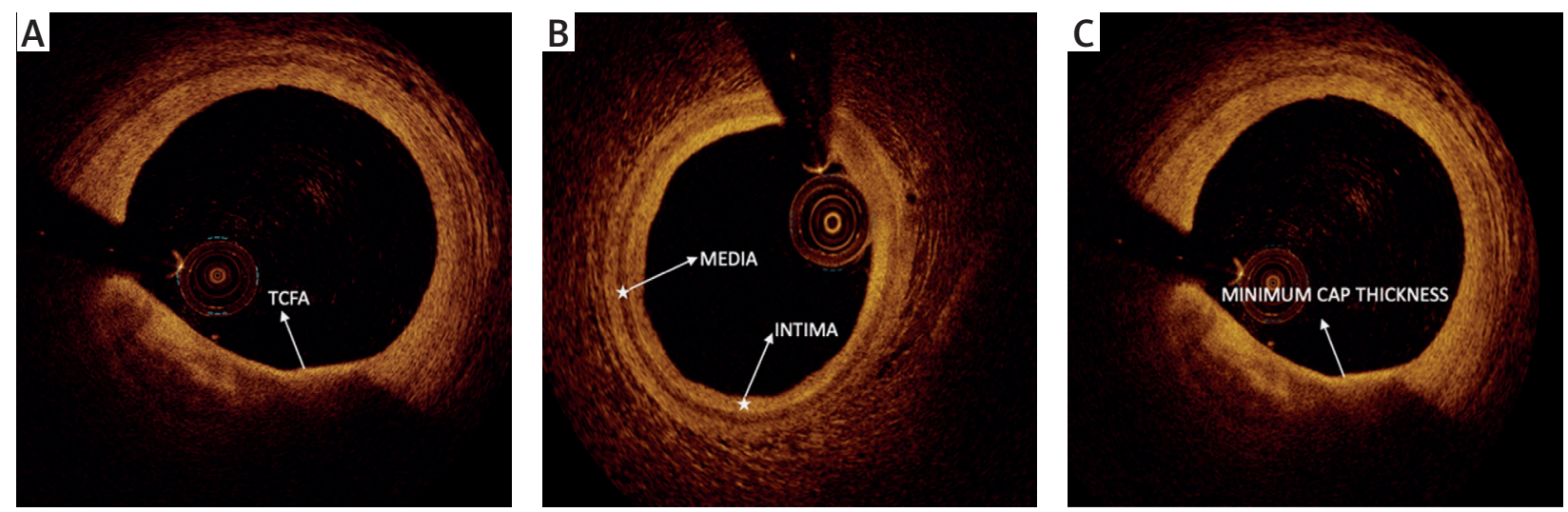

Figure 1. Cross-sectional analysis of OCT

\section{Statistical analysis}

The patients were divided into two groups according to presence of myocardial injury or type 4 a myocardial infarction following percutaneous coronary intervention: the first group (myocardial injury or type 4a myocardial infarction, $n=25$ ) and the second group (no myocardial injury or type 4 a myocardial infarction, $n=49$ ). Continuous and categorical variables were expressed as mean \pm standard deviation and percentages, respectively. Group means for continuous variables were compared using the Mann-Whitney $U$ test and independent sample $t$-test. Categorical variables were compared using the $\chi^{2}$ test or Fisher's exact test, as appropriate. In order to perform the univariate comparison between IGF-1 level with reference intimal thickness, reference medial thickness and plaque fibrous cap thickness, Spearman and Pearson correlation analysis was applied. To determine the independent predictors of myocardial injury or type 4 a infarction, univariate and multivariate logistic regression analysis models were applied. For the multivariate logistic regression analysis model, apart from IGF-1 level, diabetes mellitus, presence of intra-plaque macrophage and cholesterol crystal, $\mathrm{HbA}_{1 \mathrm{c}}$ level, presence of stent edge dissection and post$\mathrm{PCl}$ luminal mass, and antiplatelet therapy were included. Odds ratio (OR) and 95\% confidence intervals (95\% $\mathrm{Cl}$ ) were calculated. At this scope, all variables showing a significance value $<0.05$ in correlation analysis were included in the model. In all statistical analysis, two-tailed $p$-values of $<0.05$ were considered to indicate statistical significance. ROC curve analysis were used to calculate the sensitivity and the specificity of IGF-1 for periprocedural myocardial injury and type 4a myocardial infarction. All statistical analyses were made using SPSS 11.5 (SPSS Inc, Chicago, IL, USA). The effect size (Cohen's $d$ ) and power value $(1-\beta)$ for IGF-1, compared between patients with and without periprocedural myocardial injury/type $4 a$ myocardial infarction, were calculated using the $G$ Power software (version 3.1.9.2). The $\alpha$ level used for this analysis was $<0.05$. The effect size and power value were 1.23 and 0.99 for IGF-1.

\section{Results}

We consecutively enrolled 74 patients with single de novo native coronary lesion who were treated with drug-eluting stent implantation. All patients underwent FD-OCT-guided $\mathrm{PCl}$ and were analyzed. A total of 25 (33.7\%) patients had periprocedural myocardial injury or type 4 a myocardial infarction and 49 (66.2\%) patients had no events. Of 25 patients, 7 had periprocedural myocardial injury and 18 had type 4 a myocardial infarction. Basal characteristics of both groups are described in Table I. Presence of type 2 diabetes mellitus (39\% vs. $84 \%, p<0.001)$ was more likely in the periprocedural myocardial injury or type $4 \mathrm{a}$ infarction group. $\mathrm{HbA}_{1 \mathrm{c}}$ levels (5.88 $\pm 1.10 \%$ vs. $6.90 \pm 1.63 \%, p=0.006)$ and oral antidiabetic use $(32.7 \%$ vs. $68 \%, p=0.004)$ were higher in the periprocedural myocardial injury or type $4 a$ infarction group as well. IGF-1 level $(180.5 \pm 55.6 \mathrm{ng} / \mathrm{ml}$ vs. $128.2 \pm 23.6 \mathrm{ng} / \mathrm{ml}, p<0.001)$, reference intimal (169.2 $\pm 60.7 \mu \mathrm{m}$ vs. $65.3 \pm 12.7 \mu \mathrm{m}, p<0.001)$, medial thickness $(118 \pm 21.9 \mu \mathrm{m}$ vs. $78.5 \pm 9.7 \mu \mathrm{m}, p<0.001)$ and fibrous cap thickness $(163.9 \pm 60.2 \mu \mathrm{m}$ vs. $59.1 \pm 14.2 \mu \mathrm{m}$, $p<0.001$ ) were lower in the periprocedural myocardial injury and type $4 \mathrm{a}$ infarction group. Presence of intra-plaque macrophage ( $2 \%$ vs. $32 \%, p<0.001)$ and cholesterol crystal $(4.1 \%$ vs. $44 \%, p<0.001)$ was more likely in the periprocedural myocardial injury or type $4 a$ infarction group. All of the patients had TCFA developed periprocedural myocardial injury or type $4 a$ infarction $(n=18)$. Presence of stent edge dissection ( $2 \%$ vs. $28 \%$, $p=0.001$ ) and post- $\mathrm{PCl}$ luminal mass ( $2 \%$ vs. $40 \%$, $p<0.001)$ was more likely in the periprocedural myocardial injury or infarction group. Additional stent implantation was required for only 4 patients in the periprocedural myocardial injury or type $4 \mathrm{a}$ infarction group. There was no side branch coronary occlusion after the index $\mathrm{PCl}$ procedure in either group. Procedural features and OCT parameters are shown in Table II.

IGF-1 level and reference intimal thickness, medial thickness, and fibrous cap thickness in OCT had strong correlations ( $r=0.88,0.80$ and 0.88 respectively, $p<0.001)$ 
Table I. Baseline clinical and laboratory parameters

\begin{tabular}{|c|c|c|c|}
\hline Parameter & Periprocedural injury or type $4 \mathrm{a}$ MI (-) & Periprocedural injury or type $4 \mathrm{a} \mathrm{MI}(+)$ & $P$-value \\
\hline Age [years] & $61.8(11.5)$ & $61(11.5)$ & 0.859 \\
\hline $\mathrm{BMI}\left[\mathrm{kg} / \mathrm{m}^{2}\right]$ & $27.7(3.2)$ & $27.9(3.5)$ & 0.815 \\
\hline Sex (female) & $14(28.6)$ & $10(40)$ & 0.324 \\
\hline HT & $28(57.1)$ & $17(68)$ & 0.369 \\
\hline $\mathrm{HL}$ & $33(67.3)$ & $17(68)$ & 0.955 \\
\hline Type 2 diabetes mellitus & $19(38.8)$ & $21(84)$ & $<0.001$ \\
\hline Current smoker & $28(57.1)$ & $12(48)$ & 0.458 \\
\hline Family history of CAD & $29(59.2)$ & $16(64)$ & 0.690 \\
\hline $\mathrm{HbA}_{1 \mathrm{c}} \%$ & $5.8(1.1)$ & $6.9(1.6)$ & 0.006 \\
\hline IGF-1 & $180.5(55.6)$ & $128.2(23.6)$ & $<0.001$ \\
\hline LDL cholesterol [mg/dl] & $115.5(34.7)$ & $116.7(34.9)$ & 0.819 \\
\hline HDL cholesterol [mg/dl] & $32.7(5.6)$ & $32.2(7.4)$ & 0.464 \\
\hline Triglyceride [mg/dl] & $250(72.1)$ & $245.5(59.4)$ & 0.784 \\
\hline Statin use & 39 (79.6) & $21(84)$ & 0.649 \\
\hline OAD use & $16(32.7)$ & $17(68)$ & 0.004 \\
\hline \multicolumn{4}{|l|}{ Antiplatelet choice: } \\
\hline Clopidogrel & 49 (100) & $23(92)$ & 0.046 \\
\hline Ticagrelor & $0(0)$ & $2(8)$ & \\
\hline Prasugrel & $0(0)$ & $0(0)$ & \\
\hline
\end{tabular}

BMI - body mass index, HT - hypertension, HL - hyperlipidemia, CAD - coronary artery disease, IGF-1 - insulin-like growth factor-1, LDL - low-density lipoprotein, $H D L$ - high-density lipoprotein, OAD - oral anti-diabetic.

(Table III). The level of IGF-1 was an independent predictor of periprocedural myocardial injury or type $4 \mathrm{a} \mathrm{MI}$ in univariate $(\mathrm{OR}=0.929,95 \% \mathrm{Cl}: 0.895-0.964, p<0.001)$ and multivariate regression analysis (OR $=0.757,95 \%$ $\mathrm{Cl}: 0.575-0.998, p=0.04)$. Based on ROC analysis, the best cut-off value of IGF-1 for predicting periprocedural myocardial injury or type 4 a myocardial infarction was $144.5 \mathrm{ng} / \mathrm{ml}$, with a maximum sensitivity of $88 \%$ and specificity of $77.6 \%$ (AUC $=0.80,95 \% \mathrm{Cl} 0.69-0.88$, $p<0.0001$ ) (Figure 2).

\section{Discussion}

The present study revealed that the plasma IGF-1 levels were significantly lower in the periprocedural myocardial injury or infarction group. Additionally, there was a strong correlation between IGF-1 levels and plaque fibrous cap thickness. To the best of our knowledge, this is the first study to describe the role of IGF-1 in plaque characteristics by assessing OCT as an intravascular imaging method. The cut-off value of IGF-1 above $145 \mathrm{ng} / \mathrm{ml}$ predicts periprocedural myocardial injury/infarction with $88 \%$ sensitivity and $77.6 \%$ specificity.
Myocardial damage after PCl defined as cardiac troponin elevation has been reported as a procedural complication. There are numerous methods such as OCT, intravascular ultrasound, and near infrared spectroscopy that are able to recognize lipid-rich plaques. However, the fibrous cap thickness according to OCT showed the highest probability to predict periprocedural MI. Additionally, OCT-verified TFCA was found to be an independent predictor for periprocedural $\mathrm{MI}$ as well as post-stent implantation such as subsequent in-stent neoatherosclerosis $[15,16]$. Similarly, previous OCT studies to predict periprocedural MI have found the cap thickness and lipid arc to be predictors of periprocedural MI [17].

IGF-1 has been associated with many physiological actions, i.e. tissue growth and development, proliferation, pro-survival/anti-ageing, lipid metabolism, anti-inflammatory, antioxidant, neuro- and hepato-protective properties. Therefore, regardless of the presence of diabetes mellitus, a high level of IGF-1 leads to plaque stability. Sirbu et al. [18] showed that low IGF-1 was associated with higher abnormality of the carotid intima media. Plaque ruptures are more likely to occur in lesions with 
Table II. Procedural features and OCT parameters. Described as $n$ and (\%)

\begin{tabular}{|c|c|c|c|}
\hline Parameter & $\begin{array}{c}\text { Periprocedural injury } \\
\text { or type } 4 a \mathrm{MI}(-)\end{array}$ & $\begin{array}{l}\text { Periprocedural injury } \\
\text { or type } 4 \mathrm{a} \mathrm{MI}(+)\end{array}$ & $P$-value \\
\hline Vessel: & & & 0.514 \\
\hline LAD & $24(49)$ & $14(56)$ & \\
\hline$C x$ & $13(26.5)$ & $6(24)$ & \\
\hline RCA & $12(24.5)$ & $5(20)$ & \\
\hline Predilatation (8 atm) & $5(10)$ & $2(9)$ & 1 \\
\hline Additional stent use & $0(0)$ & $4(16)$ & 0.004 \\
\hline Postdilatation with NC balloon (18 atm) & $12(24)$ & $3(12)$ & 1 \\
\hline Side branch occlusion after $\mathrm{PCl}$ & $0(0)$ & $0(0)$ & 1 \\
\hline No-reflow after $\mathrm{PCl}$ & $0(0)$ & $1(0.04)$ & 0.16 \\
\hline Stent diameter [mm] & $2.8(0.37)$ & $2.9(0.4)$ & 0.361 \\
\hline Stent length $[\mathrm{mm}]$ & $23.3(7)$ & $22.8(7.2)$ & 0.816 \\
\hline Wall motion abnormalities on echo after PCI & $0(0)$ & $1(4)$ & 0.162 \\
\hline \multicolumn{4}{|l|}{ Plaque type at OCT: } \\
\hline Fibrocalcific & $12(24.5)$ & $5(20)$ & 0.230 \\
\hline Lipid rich & $21(42.9)$ & $18(72)$ & \\
\hline Fibrous & $16(32.7)$ & $2(8)$ & \\
\hline Lipid length at OCT [mm] & $18.2(7)$ & $17.9(6.8)$ & 0.873 \\
\hline Mean lipid at OCT $\left[{ }^{\circ}\right]$ & $250.1(84.5)$ & $254.8(80.7)$ & 0.752 \\
\hline Lipid volume index at OCT & $4481.5(2243.8)$ & $4523.12(2217.1)$ & 0.841 \\
\hline MLD at baseline OCT [mm] & $1.4(0.4)$ & $1.4(0.3)$ & 0.904 \\
\hline MLA at baseline OCT [mm] & $1.7(0.7)$ & $1.6(0.7)$ & 0.810 \\
\hline RLD at baseline OCT [mm] & $2.8(0.4)$ & $2.9(0.4)$ & 0.317 \\
\hline RLA at baseline OCT [mm] & $6.3(1.7)$ & $6.8(1.9)$ & 0.285 \\
\hline Area stenosis at baseline OCT (\%) & $71.5(11.2)$ & $74.2(10.1)$ & 0.303 \\
\hline Presence of microvessels at baseline OCT & $12(24.5)$ & $2(8)$ & 0.089 \\
\hline Presence of cholesterol crystals at baseline OCT & $2(4.1)$ & $11(44)$ & $<0.001$ \\
\hline Presence of macrophage at baseline OCT & $1(2)$ & $8(32)$ & $<0.001$ \\
\hline Presence of calcified nodules at baseline OCT & $5(10.2)$ & $2(8)$ & 0.761 \\
\hline Ruptured plaque at baseline OCT & $2(4.1)$ & $0(0)$ & 0.309 \\
\hline TCFA at baseline OCT & $0(0)$ & $18(72)$ & $<0.001$ \\
\hline Thrombus at baseline OCT & $2(4.1)$ & $0(0)$ & 0.309 \\
\hline Spotty calcification at baseline OCT & $9(18.4)$ & $8(32)$ & 0.190 \\
\hline Reference intimal thickness at baseline OCT [um] & $169.2(60.8)$ & $65.4(12.7)$ & $<0.001$ \\
\hline Reference medial thickness at baseline OCT [um] & $118.1(22)$ & $78.5(9.7)$ & $<0.001$ \\
\hline Minimum cap thickness of plaque at baseline OCT [ $\mu \mathrm{m}]$ & $163.9(60.2)$ & $59.2(14.2)$ & $<0.001$ \\
\hline Stent edge dissection at OCT & $1(2)$ & $7(28)$ & 0.001 \\
\hline Luminal mass after $\mathrm{PCl}$ at $\mathrm{OCT}$ & $1(2)$ & $10(40)$ & $<0.001$ \\
\hline
\end{tabular}

NC - non-compliant, $P C l$ - percutaneous coronary intervention, OCT - optical coherence tomography, MLD - minimal lumen diameter, MLA - minimal lumen area $R L D$-reference lumen diameter, RLA - reference lumen area, TCFA - thin cap fibroatheroma. 
Table III. Correlation between IGF-1 level and reference intimal, medial and plaque minimum cap thickness

\begin{tabular}{lc} 
Parameter & IGF-1 level \\
\hline Reference intimal thickness & $r=0.88$ \\
& $p<0.001$ \\
\hline Reference medial thickness & $r=0.80$ \\
& $p<0.001$ \\
\hline Plaque minimum cap thickness & $r=0.88$ \\
& $p<0.001$
\end{tabular}

thin fibrous caps, high concentrations of lipid-filled macrophages, and those with large necrotic cores. Apoptosis and necrosis play a very important role in this condition by forming the necrotic core. Additionally, apoptosis of smooth muscle and macrophages makes lesion regression likely unfavorable. For this reason, IGF-1 deficiency has a central role in this whole process, as it is one of the most potent antiapoptotic, antioxidant and cytoprotective factors known today. Moreover, IGF-1 is also known to have vasodilator actions [19].

IGF-1 regulates cell proliferation, apoptosis, migration and differentiation, so it is a potent mitogen factor for vascular smooth cells to stimulate migration and antiapoptotic effects of VSMC [20]. Thus, the reduction of levels leads to plaque instability. An experimental study showed that IGF-1 in atherosclerotic plaques may have a role in preventing plaque instability by modulating smooth muscle cell turnover and phenotype [21]. In furtherance of this finding, the PRIME study, which evaluated the relationship between IGF-1 and occurrence of acute coronary syndromes, showed that high levels of IGF-1 appear as a negative, independent predictor of occurrence of acute coronary syndromes at 5 years [22]. Thus, according to this study, IGF-1 might have a role in plaque denudation and destabilization.

In our study, plaque fibrous cap thickness was lower in the periprocedural myocardial injury/infarction group and all patients with TCFA had periprocedural myocardial injury/infarction. Additionally, IGF-1 levels were found to be lower in these patients.

Although this study for the first time examined the relationship between IGF-1 and periprocedural myocardial injury/infarction following $\mathrm{PCl}$, it also has limitations. It was a single-centered study and the number of patients was small. We only measured free IGF-1 and measurements of IGF-binding proteins (IGF-BP) were not available. Future studies that include not only IGF-1 levels, but also circulating IGF-BPs, will allow a better understanding of the mechanisms responsible for the associations observed in the present study.

\section{Conclusions}

The results from this study indicate that low IGF-1 levels are associated with plaque instability assessed

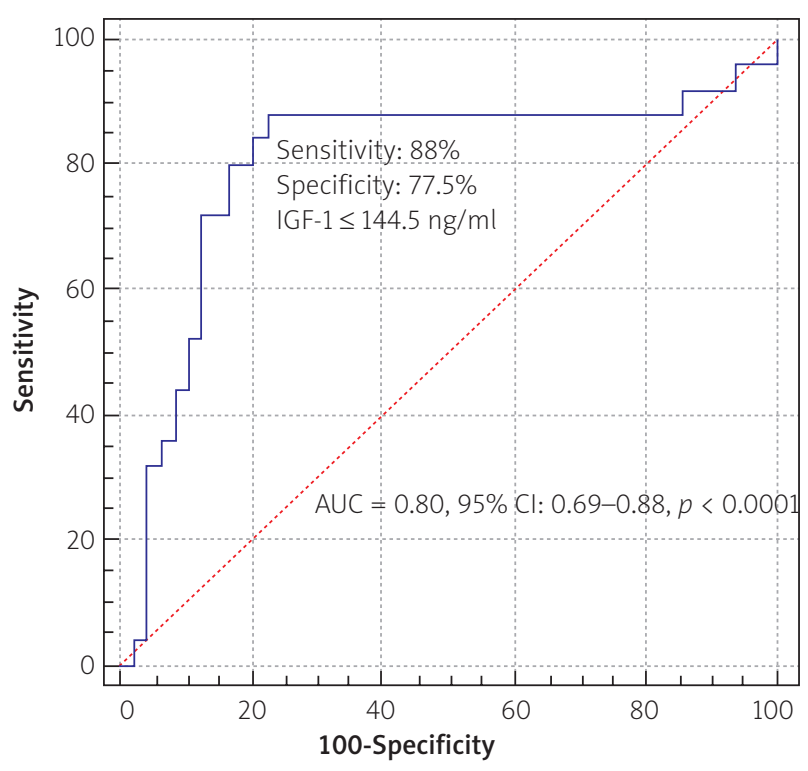

Figure 2. ROC analysis

by OCT. There was a strong correlation between IGF-1 level and plaque fibrous cap thickness in OCT. Patients with high IGF-1 levels had a thicker plaque fibrous cap, i.e. more stable plaque. Therefore, IGF-1 levels may play a protective role in the myocardial injury after coronary stent placement. Low IGF-1 levels may identify patients who are at increased risk for periprocedural myocardial injury/infarction. This study may elucidate the mechanism responsible for periprocedural myocardial injury/ infarction. In the presence of a low level of IGF-1 prior to elective $\mathrm{PCl}$, more potent antiplatelet therapy (prasugrel, ticagrelor, glycoprotein IIb/IIla inhibitors) might be of value in preventing periprocedural MI. Further experimental and prospective clinical studies are needed to demonstrate the link between the plaque characteristics and IGF-1.

\section{Conflict of interest}

The authors declare no conflict of interest.

\section{References}

1. Liou K, Jepson N, Kellar P, Ng B, et al. Prognostic significance of peri-procedural myocardial infarction in the era of high sensitivity troponin: a validation ofthe joint ACCF/AHA/ESC/WHF universal definition of type 4 amyocardial infarction with high sensitivity troponin T. Heart Lung Circ 2015; 24: 673-81.

2. Von der Thüsen JH, Borensztajn KS, Moimas S, et al. IGF-1 has plaque-stabilizing effects in atherosclerosis by altering vascular smooth muscle cell phenotype. Am J Pathol 2011; 178: 924-34.

3. Jing Z, Hou X, Wang Y, et al. Association between insulin-like growth factor-1 and cardiovascular disease risk: evidence from a meta-analysis. Int J Cardiol 2015; 198: 1-5.

4. Hansson HA, Jennische E, Skottner A. Regenerating endothelial cells express insulin-like growth factor-1 immunoreactivity after arterial injury. Cell Tissue Res 1987; 250: 499-505. 
5. Jones DA, Rathod KS, Koganti S, et al. Angiography alone versus angiography plus optical coherence tomography to guide percutaneous coronary intervention: outcomes from the pan-london PCI cohort. JACC Cardiovasc Interv 2018; 11: 1313-21.

6. Cilingiroglu $M$, Oh JH, Sugunan $B$, et al. Detection of vulnerable plaque in a murine model of atherosclerosis with optical coherence tomography. Catheter Cardiovasc Interv 2006; 67: 915-23.

7. Kini AS, Motoyama S, Vengrenyuk Y, et al. Multimodality intravascular imaging to predict periprocedural myocardial infarction during percutaneous coronary intervention. JACC Cardiovasc Interv 2015; 8: 937-45.

8. Thygesen K, Alpert JS, Jaffe AS, et al.; ESC Scientific Document Group. Fourth universal definition of myocardial infarction (2018). Eur Heart J 2019; 40: 237-69.

9. Tearney GJ, Regar E, Akasaka T, et al.; International Working Group for Intravascular Optical Coherence Tomography (IWGIVOCT). Consensus standards for acquisition, measurement, and reporting of intravascular optical coherence tomography studies: a report from the International Working Group for Intravascular Optical Coherence Tomography Standardization and Validation. J Am Coll Cardiol 2012; 59: 1058-72.

10. Gonzalo N, Serruys PW, Okamura T, et al. Optical coherence tomography assessment of the acute effects of stent implantation on the vessel wall: a systematic quantitative approach. Heart 2009; 95: 1913-9.

11. Inoue T, Shinke T, Otake H, et al. Impact of strut-vessel distance and underlying plaque type on the resolution of acute strut malapposition: serial optimal coherence tomography analysis after everolimus-eluting stent implantation. Int J Cardiovasc Imaging 2014; 30: 857-65.

12. Kato K, Yonetsu T, Kim SJ, et al. Nonculprit plaques in patients with acute coronary syndromes have more vulnerable features compared with those with non-acute coronary syndromes: a 3-vessel optical coherence tomography study. Circ Cardiovasc Imaging 2012; 5: 433-40.

13. Prati F, Guagliumi G, Mintz GS, et al.; Expert's OCT Review Document. Expert review document part 2: methodology, terminology and clinical applications of optical coherence tomography for the assessment of interventional procedures. Eur Heart J 2012; 33: 2513-20.

14. Afolabi A, Mustafina I, Zhao L, et al. Does spotty calcification attenuate the response of nonculprit plaque to statin therapy? A serial optical coherence tomography study. Catheter Cardiovasc Interv 2018; 91: 582-90.

15. Porto I, Di Vito L, Burzotta F, et al. Predictors of periprocedural (type IVa) myocardial infarction, as assessed by frequency-domain optical coherence tomography. Circ Cardiovasc Interv 2012; 5: 89-96.

16. Sanuki Y, Sonoda S, Muraoka Y, et al. Contribution of poststent irregular protrusion to subsequent in-stent neoatherosclerosis after the second-generation drug-eluting stent implantation. Int Heart J 2018; 59: 307-14.

17. Tanaka A, Imanishi T, Kitabata $\mathrm{H}$, et al. Lipidrich plaque and myocardial perfusion after successful stenting in patients with non-ST-segment elevation acute coronary syndrome: an optical coherence tomography study. Eur Heart J 2009; 30: 1348-55.

18. Sirbu A, Nicolae H, Martin S, et al. IGF-1 and insulin resistance are major determinants of common carotid artery thickness in morbidly obese young patients. Angiology 2016; 67: 259-65.

19. Aguirre GA, González-Guerra JL, Espinosa L, Castilla-Cortazar I. Insulin-like growth factor 1 in the cardiovascular system. Rev Physiol Biochem Pharmacol 2018; 175: 1-45.
20. Delafontaine P, Song YH, Li Y. Expression, regulation, and function of IGF-1, IGF-1R, and IGF-1 binding proteins in blood vessels. Arterioscler Thromb Vasc Biol 2004; 24: 435-44.

21. von der Thüsen JH, Borensztajn KS, Moimas S, et al. IGF-1 has plaque-stabilizing effects in atherosclerosis by altering vascular smooth muscle cell phenotype. Am J Pathol 2011; 178: 924-34.

22. Ruidavets JB, Luc G, Machez E, et al. Effects of insulin-like growth factor 1 in preventing acute coronary syndromes: the PRIME study. Atherosclerosis 2011; 218: 464-9. 